\title{
DIRECT AND INVERSE STRONG-TYPE INEQUALITIES FOR JACKSON-MATSUOKA POLYNOMIALS ON THE SPHERE
}

\author{
Guo Feng And YuAn Feng
}

Abstract. The direct and inverse strong-type inequalities are established for the best approximation by Jackson-Matsuoka polynomials in the $L_{p}$ space on the unit sphere of $\mathbb{R}^{d}$ which with the help of the relation between K-functionals and modulus of smoothness of sphere.

Mathematics subject classification (2010): 41A25, 41A35, 41A63.

Keywords and phrases: Jackson-Matsuoka polynomials, best approximation, modulus of smoothness, $\mathrm{K}$-functionals, spherical means.

\section{REFERENCES}

[1] H. Berens, P. L. Butzer, S. Pawelke, Limitierungsverfahren von Reihen mehrdimensionaler Kugelfunktionen und deren Saturationsverhalten, Publ. Res. Inst. Math. Sci. Ser. A. 4 (1968), 201268.

[2] E. Belinsky, F. Dai, Z. DitZian, Multivariate approximating averages, J Approx. Theory $\mathbf{1 2 5}$ (2003), 85-105.

[3] W. Z. Chen, Approximation Theory of Operators, Xiamen Univ. Publishing House, 1989. (In Chinese)

[4] Z. DitZian, Jackson-type inequality on the sphere, Acta Math. Hungar. 102, 1-2 (2004), 1-35.

[5] Z. Ditzian, K. Runovs KiI, Averages on caps of $\mathbb{S}^{d-1}$, J. Math. Anal. Appl. 248 (2000), 260-274.

[6] W. Freedden, T. Gervens, M. Schreiner, Constructive approximation on the sphere, New York: Oxford University Press Inc., 1998.

[7] K. Y. WANG, L. Q. LI, Harmonic analysis and approximation on the unit sphere [M], Beijing: Science Press, 2000.

[8] Y. MatsuoKa, On the approximation of functions by some singular integrals, Tohoku Math. J. 18 (1966), 13-43.

[9] C. MüLLER, Spherical harmonics, Lecture Notes on Mathematics, Vol. 17, Berlin: Springer-Verlag, 1971.

[10] S. PAWELKE, Über Approximationsordnung bei Kugelfunktionen und algebraischen Polynomen, Tohoku Math. J. 24 (1972), 473-486.

[11] S. RiemenschneIder, K. Y. WAng, Approximation theorems of Jackson type on the sphere, Adv. in Math. (In Chinese) 24, 2 (1995), 184-186.

[12] M. E. Stein, G. Weiss, Introduction of functions of real variable, Princeton: Princeton University Press, 1971. 